\title{
Sawtooth-Like Graphene Nanoribbon
}

\author{
Xiaojun Wu and Xiao Cheng Zeng $(\bowtie)$ \\ Department of Chemistry and Nebraska Center for Materials and Nanoscience, University of Nebraska-Lincoln, Lincoln, NE 68588, \\ USA
}

Received: 2 April 2008 / Revised: 24 April 2008 / Accepted: 25 April 2008

(C)Tsinghua Press and Springer-Verlag 2008

\begin{abstract}
Motivated by recent successful synthesize of segmented graphene nanoribbons (GNRs) with junctions, we explore electronic properties of a novel form of GNR with sawtooth-like structure using the density-functional theory method. It is found that the unique edge structures of the sawtooth-like GNR induce richer band-gap features than the straight GNR counterpart with either armchair or zigzag edges. The effect of external electric field on the electronic properties of the sawtooth-like GNR is also studied. The theoretical results may be useful for designing GNR-based field-effect transistors.
\end{abstract}

\section{KEYWORDS}

Graphene nanoribbons, sawtooth-like GNR, straight GNR, edge effect

Graphene, a single layer of graphite, has attracted considerable attention recently due to its intriguing physical properties and potential application in nanoelectronics [1-5]. By patterning and cutting a graphene sheet into one-dimensional nanoribbons, both experimental and theoretical studies have shown that the electronic band gap of the graphene can be opened up. Moreover, the band gap of the graphene nanoribbon (GNR) is dependent on its width and crystallographic orientation [6-15], rendering graphene-based band-structure engineering and nanoelectronic device possible [14-20]. It is also reported that a finite graphene fragment with special edges can exhibit giant spin moments [1621]. Several theoretical studies have predicted that half-metallicity may be realized in GNR either by applying an external in-plane electric field or by chemically functionalizing the zigzag-edge of GNR with different molecular groups (such as $\mathrm{H}, \mathrm{COOH}$,
$\mathrm{OH}, \mathrm{NO}_{2}, \mathrm{NH}_{3}, \mathrm{CH}_{3}$, etc.) [22-25]. The half-metallic GNR may find application in spintronics.

Recently, Dai and co-workers have successfully synthesized sub-10 nm GNRs with ultra-smooth edges by using a chemical approach [7]. Straight GNRs with zigzag, armchair, or mixed edges are proven to be semiconducting by the experiment. Moreover, segmented GNRs containing lattice-defined graphene junctions have been produced with chemical method, where two segments formed a special angle of 120 degree. These segmented nanoribbons, when tailored together, will form a novel sawtooth-like structure. Motivated by the major advance in GNR synthesis, we have investigated infinitely long segmented GNRs with sawtooth-like structure using the first-principles method. The effect of external in-plane electric field on the electronic properties of the sawtooth-like GNRs is also studied.

The first-principle calculations were carried out

Address correspondence to xczeng@phase2.unl.edu 
using linear combination of atomic orbital densityfunctional theory (DFT) method implemented in DMol3 package [26-28]. The generalized gradient approximation (GGA) in the Perdew-Burke-Ernzerhof (PBE) form as well as an all-electron double numerical basis set with polarized function (DNP) were chosen for the spin-unrestricted DFT calculation [29]. The real-space global cutoff radius was set to be $3.70 \AA$. To simulate sawtooth-like nanoribbon, a tetragonal supercell was adopted. The nearest distance between two neighboring GNR is greater than $30 \AA$. The $k$-point sampling was employed using the Monkhorst-Pack scheme with spacing of $0.04 \AA^{-1}$ [30]. The structures were fully optimized without any constrain, and the forces on atoms were less than $0.05 \mathrm{eV} / \AA$ after geometric optimization.

The structure of a sawtooth-like GNR can be characterized by two integers $(w, l)$ as shown in Fig. 1(a). The first integer denotes the width of the

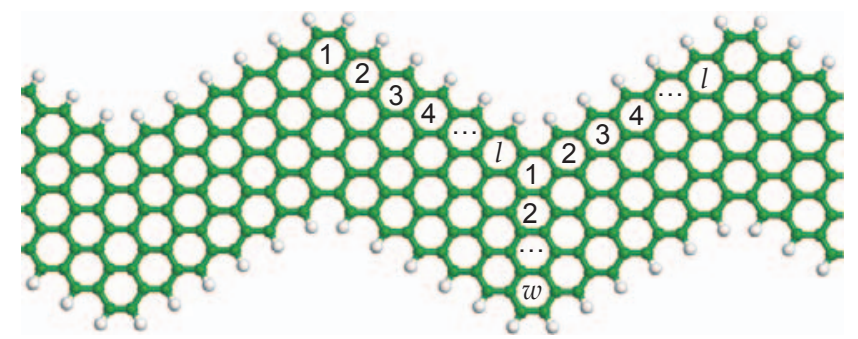

(a)

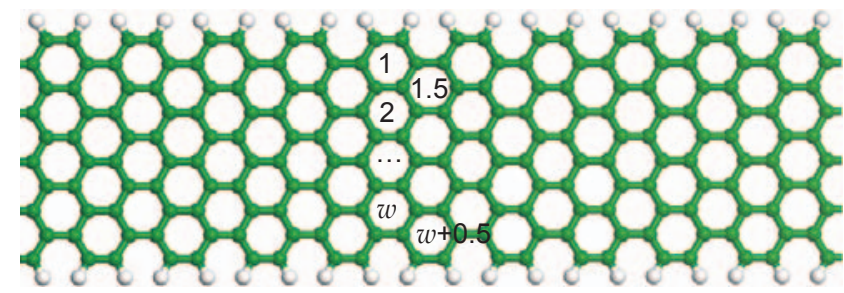

(b)

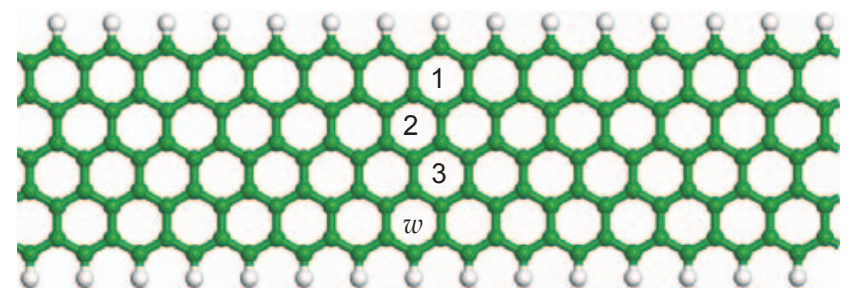

(c)

Figure 1 (a) The optimized structure of a sawtooth-like GNR with $w=4$ and $l=6$. (b) and (c) are the optimized structures of straight GNRs with armchair and zigzag edges, respectively. The green and white spheres represent carbon and hydrogen atoms, respectively nanoribbon while the second integer describes its periodic length. All edge atoms are passivated with hydrogen atoms to avoid the dangling bonds. We investigated sawtooth-like GNRs with $w$ ranging from 1 to 5 and $l$ ranging from 3 to 6 . For the purpose of comparison, straight GNRs with both armchair and zigzag edges, as shown in Figs. 1(b) and (c), were also studied. The sawtooth-like GNRs have zigzag edges only.

First, we calculated electronic properties of straight GNRs. In Fig. 2, the electronic band structures and band gaps of the GNRs with various $w$ are presented. Clearly, the electronic band structures are sensitive to the edge structure of the nanoribbons.

Consistent with previous studies, all straight

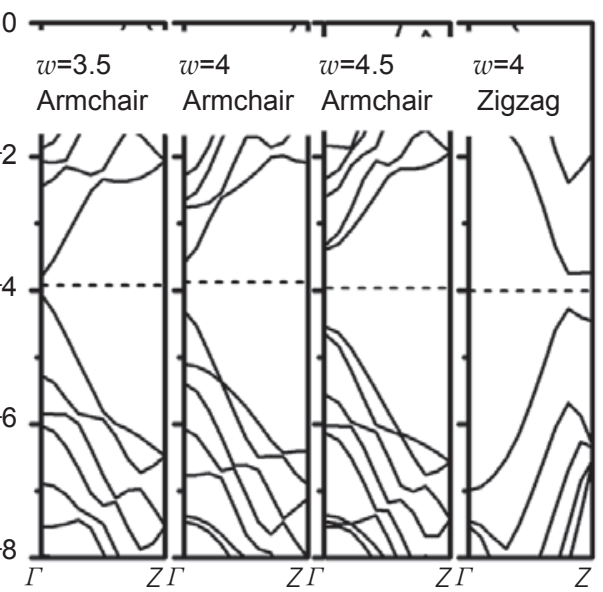

(a)

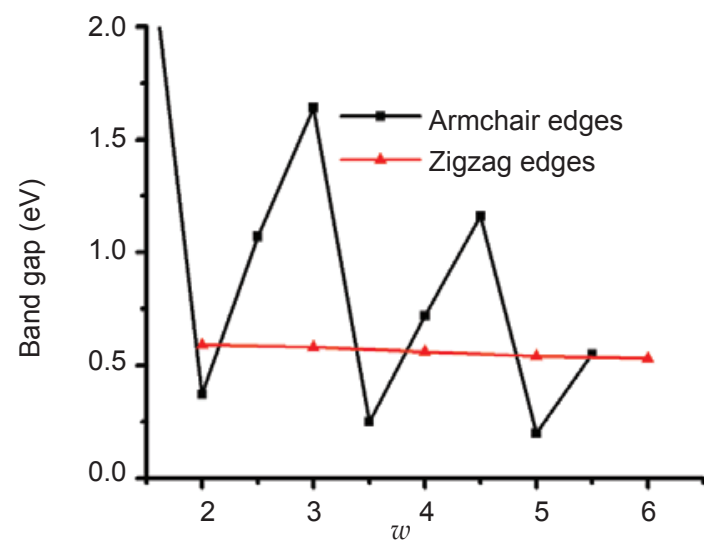

(b)

Figure 2 (a) The electronic band structures and (b) calculated band gap versus ribbon width $w$ for straight GNRs. The Fermi level is plotted with the dotted line. $\Gamma$ and $Z$ correspond the $(0,0,0)$ and $(0,0,5) k$ points in the first Brillouin zone, respectively 
GNRs are semiconducting. Two distinct features can be seen by concerning the band gap. The band gap of GNR with zigzag edges slightly decreases with increasing the width $w$, while that of GNR with armchair edges varies periodically as a function of $w$. Note that previous theoretical studies have shown that the band gap of narrow GNR with armchair edges generally opens up due to the quantum confinement as well as the edge effect. The band gap of GNR with zigzag edges is due to distinct sub-lattice potential induced by edge magnetization [12]. The oscillatory band gap for GNR with armchair edges can be explained by the Fermi wavelength in the direction normal to the ribbon direction [13]. Our results of the band structures are consistent with previous theoretical studies [8-14].

Like the straight GNRs, the calculated electronic band structures of the sawtooth-like GNRs also show semiconducting characteristics with direct band gap (Fig. 3(a)). More interestingly, even though the sawtooth-like GNRs have zigzag edges (Fig. 1(a)), their band gaps show similar oscillatory behavior as those of straight GNRs with armchair edges (Fig. 3(b)), which depend on the width w. However, for most nanoribbons, their band gap reduces monotonically with increasing the periodic length $l$ (Fig. 3(c)). The band gaps of nanoribbons with $w=1,2$, or 4 reduce much rapidly, whereas those of nanoribbons with $w=3$ or 5 reduce gradually. For $(2, l)$ sawtooth-like nanoribbons, the band gap approaches to zero rapidly as the $l$ increases. These results show that the band gaps of the sawtooth-like GNRs are much more sensitive to their geometric structures, which can be exploited for band-structure engineering.

The richer band-gap behavior for the sawtoothlike GNRs stems mainly from their unique edge structures. We plotted the spin charge density profiles of straight GNRs with zigzag and armchair edges and those of sawtooth-like GNRs in Fig. 4. It is known that the lattice sites of a graphene can be classified into two sub-lattices. The spin is polarized in one direction on one sub-lattice and in opposite direction on the other. Thus, the straight GNR with zigzag edges has two opposite spins on the opposing edges (Fig. 4(a)), which results in a finite band gap.

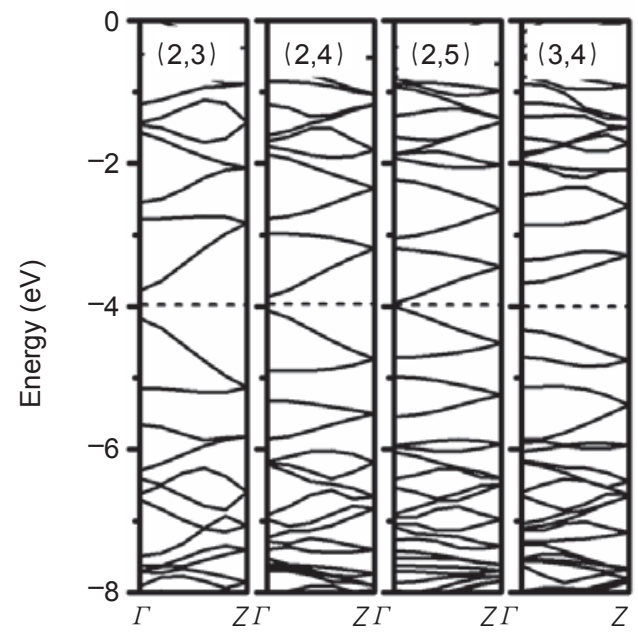

(a)

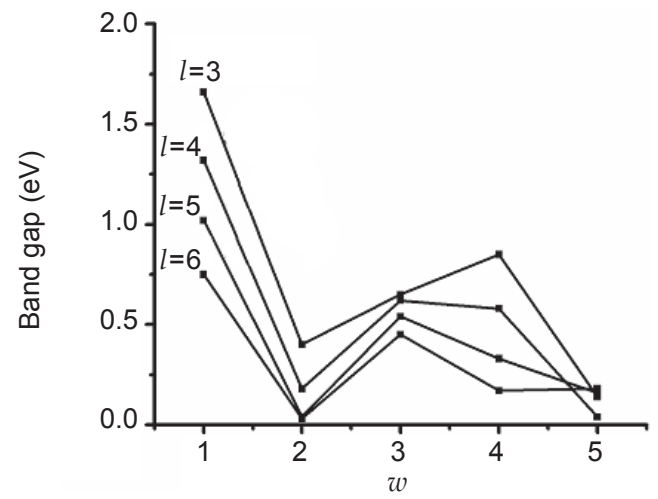

(b)

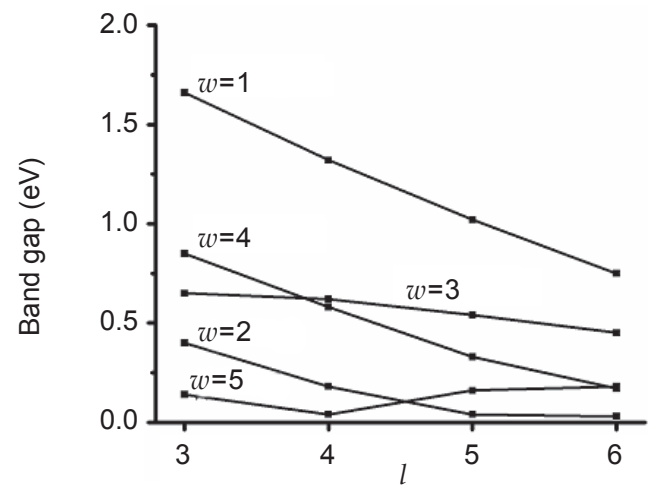

(c)

Figure 3 (a) The electronic band structures of several sawtooth-like GNRs with various $w$ and $l$. The band gaps versus (b) the width $w$ and (c) the periodic length $l$

However, this effect is negligible in the straight GNR with armchair edges because the nearest-neighbor atoms on one edge have opposite spin directions. As shown in Fig. 4(e), the spin density is very small. Hence, the ground state of the armchair GNR can be viewed as spin-restricted. We performed a spin- 


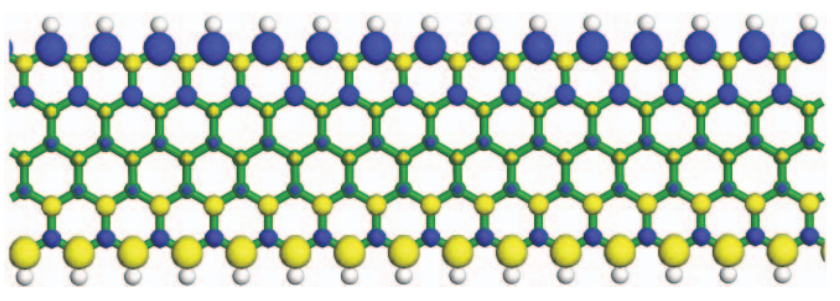

(a)

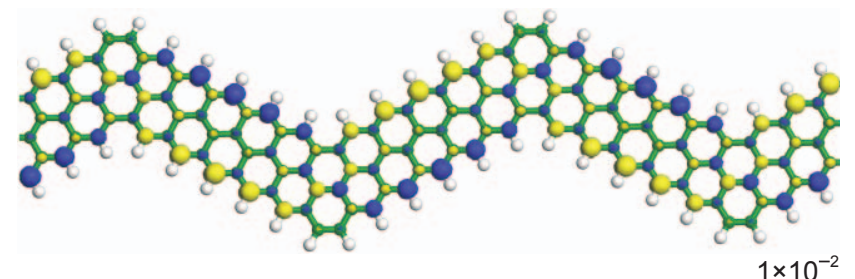

(b)

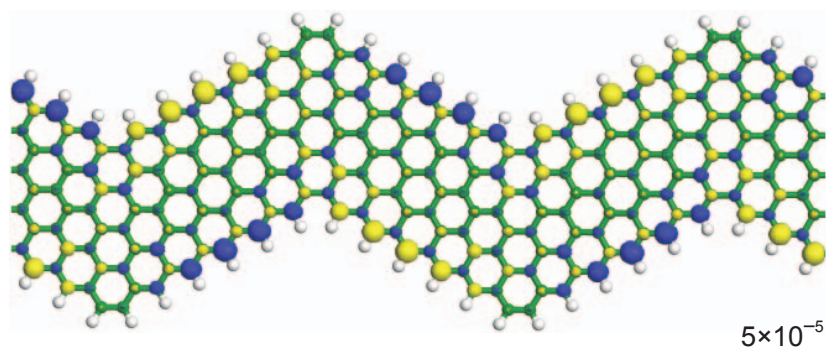

(c)

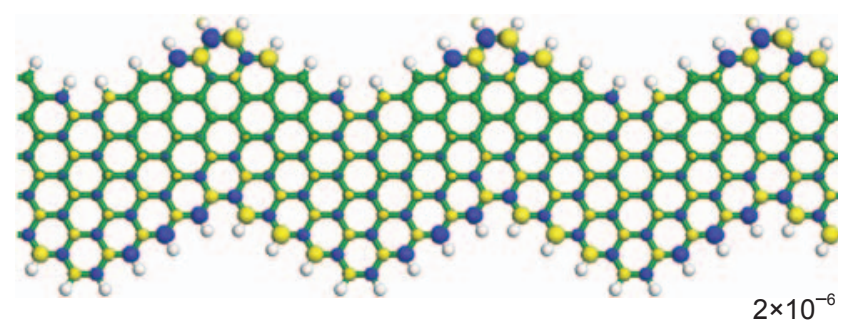

(d)

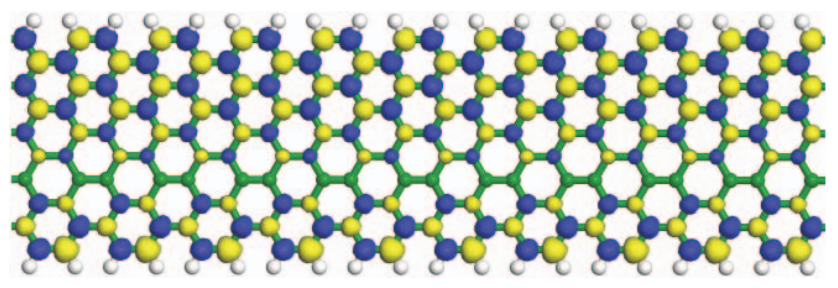

(e)

$5 \times 10^{-7}$

Figure 4 The spin charge density profiles of (a) a straight GNR with zigzag edges, (b)-(d) sawtooth-like GNRs $(2,6),(4,6)$, and $(4,4)$, and (e) a straight GNR with armchair edges. The surface density values are given on the right in the atomic unit (a.u./ $\left.\AA^{3}\right)$. Yellow and blue spheres denote the opposite spin directions

restricted calculation with the armchair GNR and found that the energy is essentially the same as that from the spin-unrestricted calculation (the energy difference is less than $1 \mathrm{meV}$ at the present level of theory). On the other hand, the sawtooth-like GNRs with zigzag edges may be viewed as a case between the two. Atoms on the edge of two neighboring segments belong to two different sub-lattices. As such, the spin direction on one edge changes alternatively from one segment to the next. From the spin charge density profiles of $(2,6),(4,6)$ and $(4,4)$ sawtooth-like GNRs shown in Figs. 4(b) - (d), it can be seen that the spin-density value depends on the ratio $l / w$. For the $(2,6)$ GNR (Fig. $4(b)), l / w=3$ and the spin density is similar to that of straight GNR with zigzag edges. The spin density of the $(4,4)$ GNR is negligible (Fig. 4(d)), as in the case of straight GNR with armchair edges. This reduction of spin is due to the suppression of spin by edge defects [31]. This is because the corner in sawtooth-like nanoribbon may be viewed as a local armchair-edge-like defect. As $l$ decreases, the corner density increases, and thus, the degree of the spin reduction becomes larger.

To assess relative stability between sawtoothlike and straight GNRs, we calculated the average cohesive energy of three types of nanoribbons with the same $w$, as shown in Table 1. Since the calculated energy from the spin-restricted and spin-unrestricted calculation is essentially the same for the straight armchair GNR, results from the spin-unrestricted calculation are used to calculate the cohesive energy of the straight armchair GNR. We chose the unit cell of straight nanoribbon with zigzag edges as the smallest repeat unit. The average cohesive energy is defined as $E_{\text {ave }}=\left[E_{\text {total }}-n_{\mathrm{C}} E(\mathrm{C})-n_{\mathrm{H}} E(\mathrm{H})\right] / N_{\text {repeat }}($ in a unit cell), where $E$ is total energy of the system, $n_{\mathrm{C}}\left(\right.$ or $n_{\mathrm{H}}$ ) is the number of carbon (or hydrogen) atoms in a unit cell, and $N_{\text {repeat }}=2 l$ for sawtooth-like GNR, and $N_{\text {repeat }}=2$ for straight GNR with armchair edges. From Table 1, it can be seen that the average cohesive energy of sawtooth-like GNRs decreases as $l$ increases, and their values are between those of straight GNRs with armchair and zigzag edges and the same $w$. The GNR with armchair edges has the largest cohesive energy. Note that the width here is characterized by the integer of $w$ but not the actual physical width. A previous study also predicted that a straight GNR with armchair edges is more stable than the straight GNR with zigzag edges [32].

Additionally, we explored the effect of external

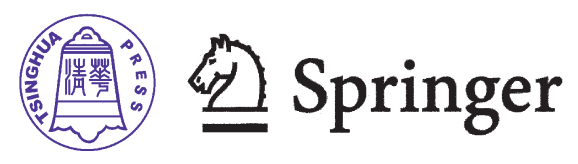


Table 1 The average cohesive energy (eV) of sawtooth-like and straight GNRs with zigzag and armchair edges

\begin{tabular}{ccccccc}
\hline \multirow{2}{*}{ GNRs } & & \multicolumn{5}{c}{ Average cohesive energy (eV) } \\
\cline { 3 - 6 } & & $w=1$ & $w=2$ & $w=3$ & $w=4$ & $w=5$ \\
\hline \multirow{2}{*}{ Sawtooth-like } & $l=3$ & -36.258 & -52.139 & -68.056 & -83.988 & -99.894 \\
GNRs & $l=4$ & -36.202 & -52.102 & -68.026 & -83.941 & -99.850 \\
& $l=5$ & -36.188 & -52.074 & -67.991 & -83.904 & -99.819 \\
& $l=6$ & -36.179 & -52.053 & -67.962 & -83.872 & -99.789 \\
\hline Straight & Zigzag edge & -36.063 & -51.959 & -67.849 & -83.744 & -99.651 \\
GNRs & Armchair edge & -36.288 & -52.212 & -68.108 & -84.033 & -99.957 \\
\hline
\end{tabular}

in-plane electric field on the electronic properties of sawtooth-like GNR. We chose the $(3,6)$ sawtooth-like GNR as a prototype model system. A uniform electric field is applied parallel to the nanoribbon's surface and normal to the ribbon direction (as the arrow shows in Fig. 5(c)). The electronic band structures and the electrostatic potential of the $(3,6)$ sawtooth-like GNR in zero field and in finite field are plotted in Fig. 5. As the field strength increases, the band structure changes, and the Fermi level is shifted downward. The band gap is also modified by the external electric field. This field effect is in stark contrast with that for the straight GNR with zigzag edges. For example, the external in-plane electric field cannot induce halfmetallicity in sawtooth-like GNR. Moreover, in the present of the electric field, the electrostatic potential distribution at two opposing edges of the sawtoothlike GNR is not symmetrical, as shown in Fig. 5(c), whereas it is quasi-symmetrical in zero field (see Fig. 5(b)). Because there exist two types of sublattice sites on one edge, the difference in electrostatic potential at the opposing edges cannot induce spin splitting but only affects the band structure and the Fermi level.

Note that in this study we only considered sawtooth-like nanoribbons with a bent angle of 120 degree between two neighboring segments. As such, the edge atoms in two neighboring segments belong to two different sub-lattices. On the other hand, if the bent angle is 60 degree, all atoms in one edge would belong to a single sub-lattice as in the case of a straight nanoribbon with zigzag edges. Hence, it is expected that sawtooth-like nanoribbons with different bent angles can have different properties.

In conclusion, we have studied stability and

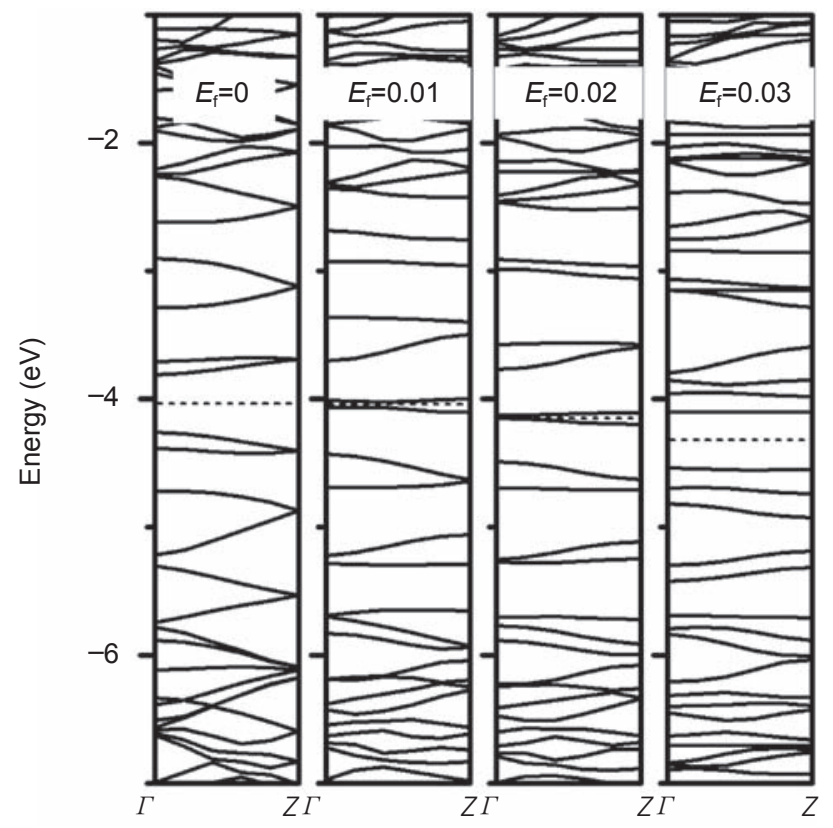

(a)

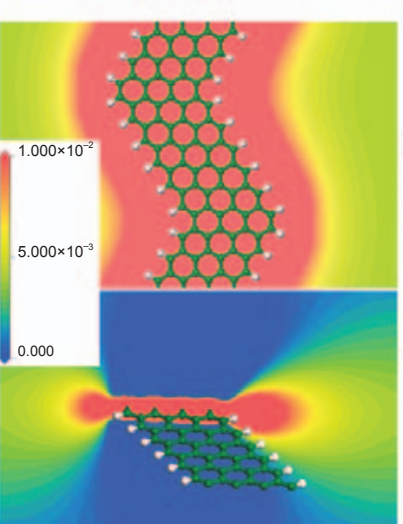

(b)

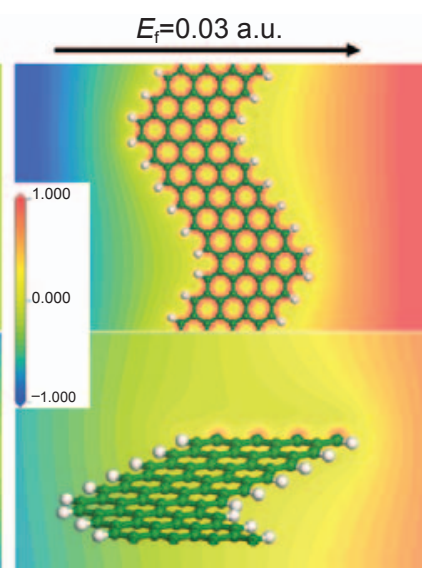

(c)
Figure 5 (a) The electronic band structures of the $(3,6)$ sawtooth-like GNR in zero and finite in-plane electric fields (The unit of the external electric field is a.u.). The Fermi level is denoted by the dotted line. The electrostatic potential distribution on the nanoribbon's surface or on the surface normal to the nanoribbon in (b) zero and (c) finite electric field $\left(E_{\mathrm{f}}\right)$ 
electronic properties of GNRs with sawtooth-like structure using the density-functional theory method. The average cohesive energy of the sawtooth-like GNR is between that of straight GNR with zigzag and armchair edges. It also decreases as the periodic length increases. The calculated electronic band structure indicates that sawtooth-like GNRs are generally semiconducting with direct band gap. The band gap is dependent on both the nanoribbon's width and periodic length. Unlike straight GNRs, an external electric field can only affect the band structure as well as the position of the Fermi level of sawtooth-like GNRs but cannot lead to half metallicity. The much richer band-gap behavior of sawtooth-like GNRs may be exploited for bandstructure engineering via tailoring geometric dimenstions of sawtooth-like GNRs.

\section{Acknowledgments}

This work is supported by grants from the DOE(DEFG02-04ER46164), NSF (CHE-0427746, CHE-0701540, and CMMI-0709333), the Nebraska Research Initiative, NSFC (\#20628304), and by the Research Computing Facility at University of NebraskaLincoln and Holland Computer Center at University of Nebraska-Omaha.

\section{References}

[1] Novoselov, K. S.; Geim, A. K.; Morozov, S. V.; Jiang, D.; Zhang, Y.; Dubonos, S. V.; Grigorieva, I. V.; Filrsov, A. A. Science 2004, 306, 666.

[2] Novoselov, K. S.; Geim, A. K.; Morozov, S. V.; Jiang, D.; Katsnelson, M. I.; Grigorieva, I. V.; Dubonos, S. V.; Firsov, A. A. Nature 2005, 438, 197.

[3] Zhang, Y. B.; Tan, Y. W.; Stormer, H. L.; Kim, P. Nature 2005, 438, 201.

[4] Berger, C.; Song, Z. M.; Li, X. B.; Wu, X. S.; Brown, N.; Naud, C.; Mayou, D.; Li, T. B.; Hass, J.; Marchenkov, A. N.; Conrad, E. H.; First, P. N.; de Heer, W. A. Science 2006, 312, 1191

[5] Berger, C.; Song, Z. M.; Li,T. B.; Li, X. B.; Ogbazghi, A. Y.; Feng, R.; Dai, Z. T.; Marchenkov, A. N.; Conrad, E. H.; First, P. N.; de Heer, W. A. J. Phys. Chem. B 2004, 108, 19912.
[6] Han, M. Y.; Özyilmaz, B.; Zhang, Y. B.; Kim, P. Phys. Rev. Lett. 2007, 98, 206805.

[7] Li, X. L.; Wang, X. R.; Zhang, L.; Lee, S.; Dai, H. J. Science 2008, 319, 1229.

[8] Nakada, K.; Fujita, M. Phys. Rev. B 1996, 54, 17954.

[9] Miyamoto, Y. Phys. Rev. B 1999, 59, 9858.

[10] Lee, H.; Son,Y. W.; Park, N.; Han, S.; Yu, J. Phys. Rev. B 2005, 72, 174431.

[11] Ezawa, M. Phys. Rev. B 2006, 73, 045432.

[12] Son, Y. W.; Cohen, M. L.; Louie, S. G. Phys. Rev. Lett. 2006, 97, 216803.

[13] Barone, V.; Hod, O.; Scuseria, G. E. Nano Lett. 2006, 6, 2748.

[14] Yan, Q. M.; Huang, B.; Yu, J.; Zheng, F. W.; Zang, J.; Wu, J.; Gu, B. L.; Liu, F.; Duan, W. H. Nano Lett. 2007, 7, 1469.

[15] Wang, Z. F.; Shi, Q. W.; Li, Q. X.; Wang, X. P.; Hou, J. G.; Zheng, H. X.; Yao, Y,; Chen, J. App. Phys. Lett. 2007, 91, 053109.

[16] Wang, W. L.; Meng, S.; Kaxiras, E. Nano Lett. 2008, 8, 241.

[17] Fernndez-Rossier, J.; Palacios, J. J. Phys. Rev. Lett. 2007, 99, 177204.

[18] Hod, O.; Peralta, J. E.; Scuseria, G. E. Phys. Rev. B 2007, 76, 233401.

[19] Hod, O.; Barone, V.; Scuseria, G. E. Phys. Rev. B 2008, 77, 035411.

[20] Ezawa, M. Physical Review B 2007, 76, 245415.

[21] Ezawa, M. Physica E 2008, 40, 1421.

[22] Son, Y. W.; Cohen, M. L.; Louie, S. G. Nature 2006, $444,347$.

[23] Rudberg, E.; Satek, P.; Luo, Y. Nano Lett. 2007, 7, 2211.

[24] Hod, O.; Barone, V.; Peralta, J. E.; Scuseria, G. E. Nano Lett. 2007, 7, 2295.

[25] Kan, E. J.; Li, Z. Y.; Yang, J. L.; Hou, J. G. J. Am. Chem. Soc. 2008, 130, 4224.

[26] Delley, B. J. Chem. Phys. 1990, 92, 508.

[27] Delley, B. J. Chem. Phys. 2003, 113, 7756.

[28] DMol3 is available from Accelrys. Delley.

[29] Perdew, J. P.; Burke, K.; Ernzerhof, M. Phys. Rev. Lett. 1996, 77, 3865.

[30] Monkhorst, H. J.; Pack, J. D. Phys. Rev. B 1976, 13, 5188.

[31] Huang, B.; Liu, F.; Wu, J.; Gu, B. L.; Duan, W. H. arXiv: 0708.1795v2.

[32] Koskinen, P.; Malola,S.; Häkkinen, H.arXiv: 0802.2623v1. 